\title{
Preparation of phosphostatine and phosphoepistatine from L-leucine via high diastereoselective reduction of 3-amino-2-ketophosphonates
}

\section{Ricardo De la Cruz-Cordero, Emanuel Hernándes-Núñez, Mario Fernández-Zertuche, Miguel Angel Muñoz-Hernández, and Mario Ordóñez*}

Centro de Investigaciones Químicas. Universidad Autónoma del Estado de Morelos Av. Universidad No. 1001. 62210 Cuernavaca, Mor. México.

E-mail:palacios@ciq.uaem.mx

\section{Dedicated to Professor Eusebio Juaristi on the occasion of his $55^{\text {th }}$ birthday}

(received 31 May 05; accepted 13 Jul 05; published on the web 19 Jul 05)

\begin{abstract}
The reduction of (3S)- $N, N$-dibenzylamino-2-ketophosphonate 5 derived from L-leucine with catecholborane at $-20{ }^{\circ} \mathrm{C}$ afford the (3S)- $N, N$-dibenzylamino-(2R)-hydroxyphosphonate syn-6, whereas the reduction of (3S)- $N$-benzylamino-2-ketophosphonate 9 with $\mathrm{Zn}\left(\mathrm{BH}_{4}\right)_{2}$ at $-78{ }^{\circ} \mathrm{C}$ gave the (3S)- $N$-benzylamino-(2S)-hydroxyphosphonate anti-10. The reduction in both cases was in good chemical yields and high diastereoselectivity. The hydrolysis and hydrogenolysis of syn-6 and anti-10 gave phosphostatine 12 and phosphoepistatine 13, respectively.
\end{abstract}

Keywords: Phosphostatine, phosphoepistatine, aminophosphonic acids, $\beta$-ketophosphonates, diastereoselective reduction, statine analogues

\section{Introduction}

(4S)-Amino-(3S)-hydroxy-6-methylheptanoic acid (Statine) 1, a nonproteinogenic amino acid, is a key component of pepstatin, a natural hexapeptide antibiotic isolated by Umezawa and coworkers from various species of actinomices. ${ }^{1}$ Additionally, (-)-statine $\mathbf{1}$ has attracted a lot of interest because of its potential use in the treatment of hypertension, congestive heart failure, malaria and Alzheimer's disease. For these reasons, many synthetic routes toward statine $\mathbf{1}$ and their analogues have been developed. ${ }^{2}$

On the other hand, phosphonates and phosphinates functionalized with amino and hydroxy groups have attracted considerably attention in recent years for their role in biologically relevant processes such as inhibition of rennin and HIV protease, human calpain I and their use as haptens in the development of catalytic antibodies. ${ }^{3}$ In particular, $\gamma$-amino- $\beta$-hydroxyphosphonic 
acids 2 and 3 have resulted in unique phosphate mimics with resistance to phosphatase hydrolysis. ${ }^{4}$ Additionally, the esters of the phosphonic acids 2, $\mathbf{3}$ and their analogues have been used as inhibitors of D-alanine:D-alanine ligase, ${ }^{5}$ and as excellent Leu10-Val11 replacements (LVRs) in the angiotensin II, providing a more potent inhibitory activity for rennin over porcine pepsin and bovine cathepsin D. ${ }^{6}$ As a result, numerous synthetic methods for chiral non-racemic $\beta$-amino- $\alpha$-hydroxyphosphonic 2 have been developed. ${ }^{7}$ However, to the best of our knowledge, only a few synthetic approaches to obtain optically active esters of 3-amino-2hydroxyphosphonic acid 3 have been described in the literature, which involve the reaction of the anion of methylphosphonate with $\alpha$-aminoaldehydes, ${ }^{5,6}$ and the catalytic asymmetric aminohydroxylation of $\beta, \gamma$-unsaturated phosphonates, ${ }^{7 \mathrm{f}}$ but in both methodologies the yields and diastereoselectivities remain low. Recently, Yokomatsu et al. ${ }^{8}$ described the synthesis of 3amino-2-hydroxyphosphonates with an improved diastereoselectivity via the dihydroxylation of $\beta, \gamma$-unsaturated phosphonates and the subsequent regioselective amination of their cyclic sulfates.<smiles>CC(C)C[C@H](N)[C@H](O)CC(=O)O</smiles>

Statine 1<smiles>[R]C(N)[C@H](O)P(=O)(O)O</smiles>

2<smiles>[R]C(N)[C@H](O)CP(=O)(O)O</smiles>

3

As part of our program directed to the synthesis of chiral 3-amino-2-hydroxyphosphonic acids, ${ }^{9}$ herein we describe a new methodology that affords (3S)-amino-(2R)-hydroxy-5methylhexyl-phosphonic acid (phosphostatine) and (3S)-amino-(2S)-hydroxy-5methylhexylphosphonic acid (epiphosphostatine), via high diastereoselective reduction of dimethyl (3S)-[3-( $N, N$-dibenzyl-amino)- and (3S)-[3-( $N$-benzylamino)-5-methyl-2oxohexylphosphonates, derived from L-leucine. ${ }^{10}$

\section{Results and Discussion}

(3S)- $N, N$-Dibenzylamino-2-ketophosphonate 5 was synthesized in two steps from L-leucine (Scheme 1). Thus, the first step of the synthesis was the tribenzylation of L-leucine with excess of benzyl bromide and $\mathrm{K}_{2} \mathrm{CO}_{3}$ under reflux in a mixture of $\mathrm{MeOH}: \mathrm{H}_{2} \mathrm{O}$, obtaining the corresponding (S)-N,N-dibenzylleucine benzyl ester 4 in $77 \%$ yield. ${ }^{11}$ Then, the resulting benzyl ester 4 was treated with the lithium salt of dimethyl methylphosphonate at $-78 \mathrm{oC}$ in $\mathrm{THF}$, to obtain the (3S)- $N, N$-dibenzylamino-2-ketophosphonate 5 in $98 \%$ yield. $^{12}$

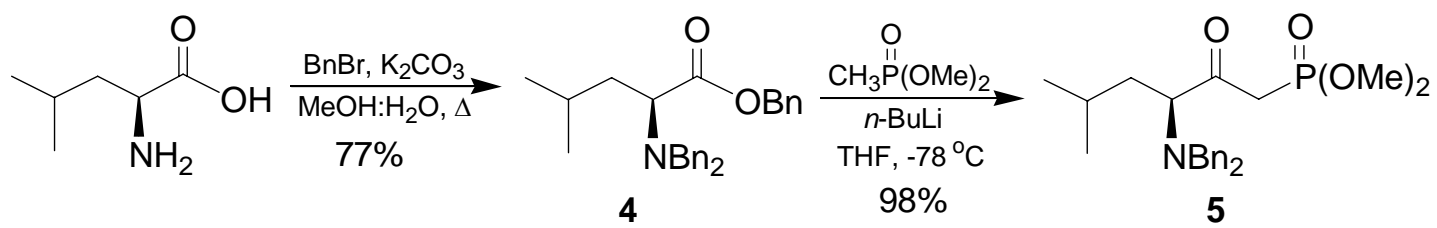

\section{Scheme 1}


Having efficiently prepared the 2-ketophosphonate 5, we turned our attention to the diastereoselective reduction of the carbonyl group to obtain the (3S)- $N$, $N$-dibenzylamino-2hydroxyphosphonates 6 and 7. The reduction was carried out using $\mathrm{NaBH}_{4}, \mathrm{Zn}\left(\mathrm{BH}_{4}\right)_{2}$ and catecholborane as reducing agents. Conditions, yields and diastereomeric ratio are summarized in Table 1.

Table 1. Reduction of 2-ketophosphonate 5 with various reducing agents

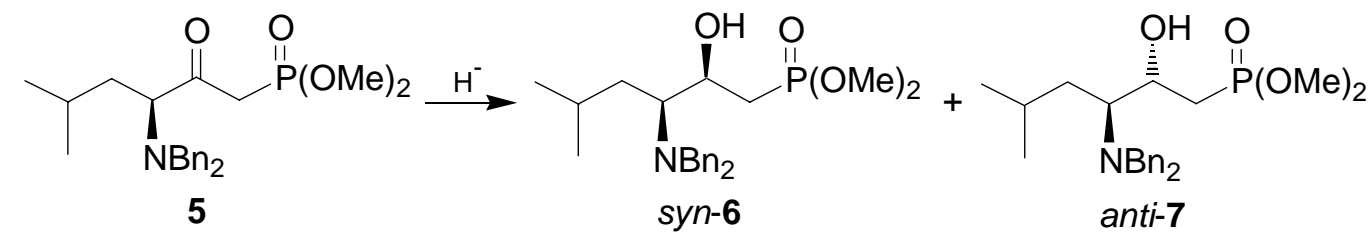

\begin{tabular}{cllcc}
\hline Entry & \multicolumn{1}{c}{ Hydride } & \multicolumn{1}{c}{ Conditions } & ${\text { Yield }(\%)^{\mathrm{a}}}$ & syn-6:anti-7 $^{\mathrm{b}}$ \\
\hline 1 & $\mathrm{NaBH}_{4}$ & $\mathrm{MeOH}, 25^{\circ} \mathrm{C}$ & 94 & $86: 14$ \\
2 & $\mathrm{Zn}\left(\mathrm{BH}_{4}\right)_{2}$ & $\mathrm{THF},-78{ }^{\circ} \mathrm{C}$ & 87 & $82: 18$ \\
3 & Catecholborane & $\mathrm{THF},-20^{\circ} \mathrm{C}$ & 85 & $>98: 2$ \\
\hline
\end{tabular}

${ }^{a}$ Chemical yield after purification by column chromatography.

${ }^{\mathrm{b}}$ Determined by ${ }^{1} \mathrm{H}$ NMR at $400 \mathrm{MHz}$ and ${ }^{31} \mathrm{P}$ NMR at $200 \mathrm{MHz}$.

As shown in Table 1, the reduction of 5 with $\mathrm{NaBH}_{4}$ at $25{ }^{\circ} \mathrm{C}$ in methanol afford the 2hydroxyphosphonates syn-6 and anti-7 with excellent chemical yield and moderate diastereoselectivity, in favor of diastereomer syn-6 (entry 1). Identical results were obtained in the reduction of 5 with $\mathrm{Zn}\left(\mathrm{BH}_{4}\right)_{2}$ (entry 2). Remarkably, when the reduction of 5 was carried out with catecholborane, only the diastereomer syn- 6 could be detected by both ${ }^{1} \mathrm{H}$ and ${ }^{31} \mathrm{P}$ NMR (entry 3). Diastereomeric ratio of the reduction of 5 was determinated by means of ${ }^{1} \mathrm{H}$ and ${ }^{31} \mathrm{P}$ NMR. In fact, in ${ }^{31} \mathrm{P}$ NMR the signal for the diastereomer syn-6 was more shielded (34.72 ppm) than that the diastereomer anti-7 $(35.37 \mathrm{ppm})$. The absolute configuration of the new stereogenic center in syn-6 and anti-7 was assigned by analogy with other (3S)- $N, N$-dibenzylamino-2hydroxyphosphonates. ${ }^{10}$

Therefore, we propose that the reduction of $\mathbf{5}$ with catecholborane took place under non-chelation control or Felkin-Ahn model, ${ }^{13}$ and that the bulkyness of the $N, N$-dibenzylamino group is sufficient to simultaneously limit the rotamer populations around the hinge bounds adjacent to the carbonyl group blocking the re face of carbonyl group and, thereby allowing the addition of hydride to take in a diastereoselective manner (Figure 1a). On the other hand, the reduction of 5 with $\mathrm{Zn}\left(\mathrm{BH}_{4}\right)_{2}$ seems to proceed in such way that the metal ions do not bind sufficiently strongly to the $N, N$-dibenzylamino and keto groups to induce chelation controlled reaction (Figure 1b). It was expected that the zinc ions would provide increased conformational control and hence higher diastereoselectivity toward the 2-hydroxyphosphonate anti-7; however, 
this is not the case, thus, the counterion does not appear to be involved on the induction of diastereoselection. This diastereofacial preference is in agreement with that reported previously for the reduction of 1-aminoalkylchloromethyl ketones, ${ }^{14}$ for the reductive amination of $\alpha$-amino ketones, ${ }^{15}$ and reduction of 1-aminoalkylchloromethyl ketimines. ${ }^{16}$

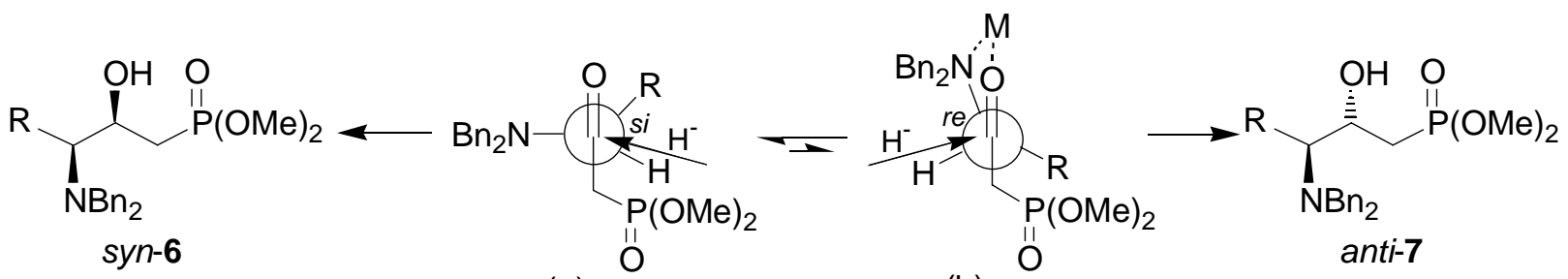
(a)

(b)

Figure 1. Reduction of 2-ketophosphonates 5: (a) non-chelation control, (b) chelation control

In order to induce the formation of anti-3-amino-2-hydroxyphosphonate, now we turned to the preparation of (3S)- $N$-benzylamino-2-ketophosphonate 9 (Scheme 2). ${ }^{17}$ Thus, the starting (S)$\mathrm{N}$-benzylleucine methyl ester $\mathbf{8}$ was prepared by treatment of the corresponding amino methyl ester hydrochloride with $\mathrm{K}_{2} \mathrm{CO}_{3}$ and benzyl bromide in acetonitrile at room temperature. Then, the methyl ester 8 was treated with the lithium salt of dimethyl methylphosphonate at $-78{ }^{\circ} \mathrm{C}$ in $\mathrm{THF}$, to afford the corresponding (3S)- $N$-benzylamino-2-ketophosphonate 9 (Scheme 2).

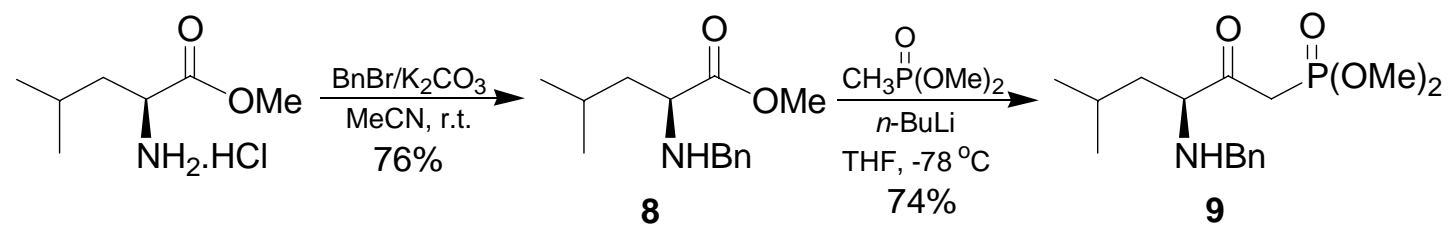

\section{Scheme 2}

Just as we have previously described, ${ }^{17}$ the reduction of 9 using $\mathrm{Zn}\left(\mathrm{BH}_{4}\right)_{2}$ at $-78{ }^{\circ} \mathrm{C}$ in THF afforded the corresponding 3- $N$-benzylamino-2-hydroxyphosphonates anti-10 and syn-11 in good chemical yield and with high diastereoselectivity, with a predominance of the desired anti product (Scheme 3).

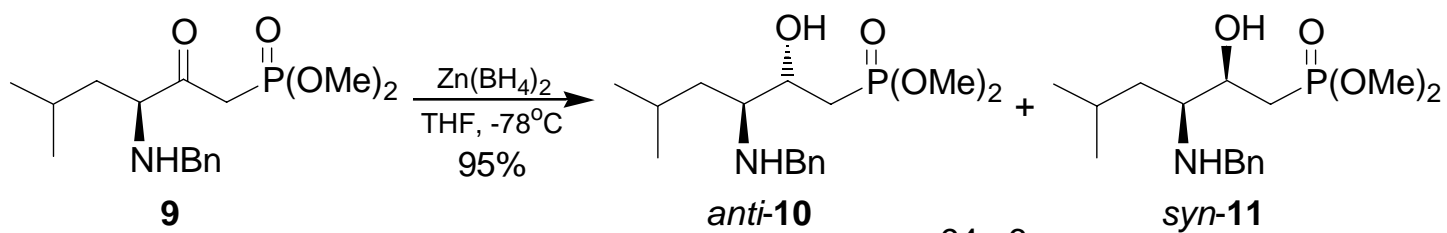

Scheme 3

Finally, the hydrolysis of 2-hydroxyphosphonates syn-6 and anti-10 with bromotrimethylsilane at room temperature ${ }^{9,18}$ afforded the corresponding 2-hydroxyphosphonic 
acid, that without any further purification was treated with palladium on carbon in methanol under hydrogen gas atmosphere at room temperature, obtaining the phosphostatine 12 and phosphoepistatine 13, in 89\% and 93\% yield, respectively, (Scheme 4).
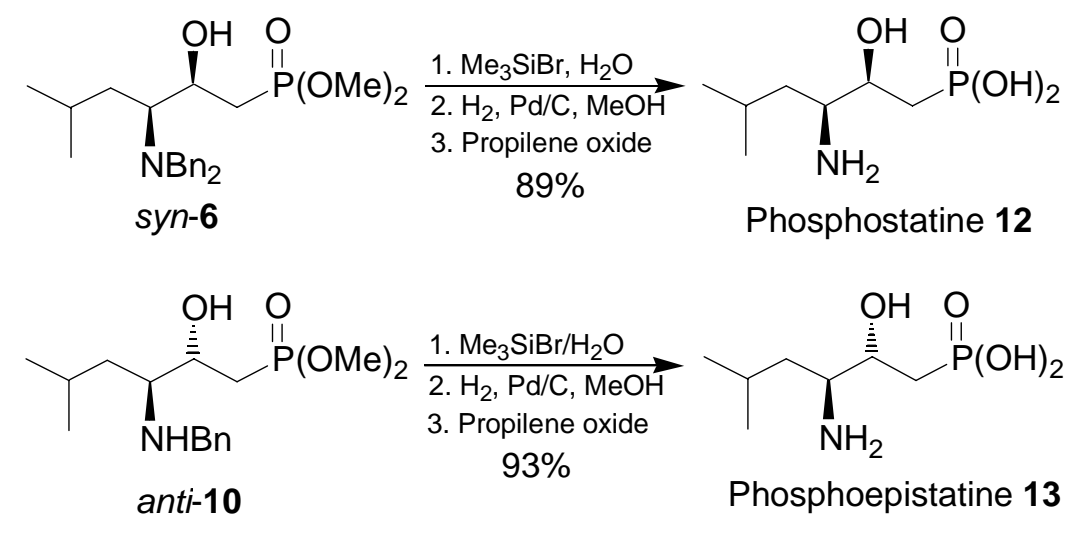

\section{Scheme 4}

In conclusion, we have found a new methodology for the preparation of phosphostatine and phosphoepistatine diastereomerically pures. Additionally, the conditions described in this paper, make this experimental operation a good and simple method to obtain the 3-amino-2hydroxyphosphonates syn and anti in high diastereoselectivity changing the protective group on the nitrogen atom of $L$-amino acid.

\section{Experimental Section}

General Procedures. Optical rotations were taken on a Perkin-Elmer 241 polarimeter in a $1 \mathrm{dm}$ tube; concentrations are given in g/100 mL. For flash chromatography, silica gel 60 (230-400 mesh ASTM, Merck) was used. ${ }^{1} \mathrm{H}$ NMR spectra were recorded on a Varian INOVA 400 (400 $\mathrm{MHz}),{ }^{13} \mathrm{C}$ NMR $(100 \mathrm{MHz})$ and ${ }^{31} \mathrm{P}$ NMR on a Varian Mercury 200 instruments. The spectra were recorded in $\mathrm{D}_{2} \mathrm{O}$ or $\mathrm{CDCl}_{3}$ solution, using TMS as internal reference. Microanalyses were registered on an Elemental VARIO EL III. MS spectra were recorded on a JEOL JMS-700.

Flasks, stirrings bars, and hypodermic needles used for the generation of organometallic compounds were dried for ca. $12 \mathrm{~h}$ at $120{ }^{\circ} \mathrm{C}$ and allowed to cool in a desiccator over anhydrous calcium sulfate. Anhydrous solvents (ethers) were obtained by distillation from benzophenone ketyl.

(S)- $N, N$-Dibenzylleucine benzyl ester $4 .^{11}$ A solution of benzyl bromide $(13.0 \mathrm{~g}, 9.1 \mathrm{~mL}$, $76 \mathrm{mmol})$ and methanol $(40 \mathrm{~mL})$ was slowly added to a mixture of L-leucine $(2.5 \mathrm{~g}, 19 \mathrm{mmol})$, $\mathrm{K}_{2} \mathrm{CO}_{3}(9.2 \mathrm{~g}, 67 \mathrm{mmol})$ in methanol-water $(5: 1,250 \mathrm{~mL})$. The reaction mixture was refluxed for $14 \mathrm{~h}$. Then, the solvent was evaporated under reduced pressure and water was added to the residue, and the resulting mixture was extracted with ethyl acetate $(3 \times 150 \mathrm{~mL})$. The combined 
organic layers were dried over $\mathrm{Na}_{2} \mathrm{SO}_{4}$, filtered and evaporated under reduced pressure. The crude product were purified by flash chromatography using hexane:AcOEt (20:1) as eluent, to give 4 as a viscous liquid $3.6 \mathrm{~g}, 77 \%$ yield. $[\alpha]_{\mathrm{D}}=-102.1\left(\mathrm{c}=3.6, \mathrm{CHCl}_{3}\right) .{ }^{1} \mathbf{H} \mathbf{~ N M R}(400 \mathrm{MHz}$, $\left.\mathrm{CDCl}_{3}\right) \delta 0.59\left(\mathrm{~d}, \mathrm{~J}=6.8 \mathrm{~Hz}, 3 \mathrm{H},\left(\mathrm{CH}_{3}\right)_{2} \mathrm{CH}\right), 0.81\left(\mathrm{~d}, \mathrm{~J}=6.8 \mathrm{~Hz}, 3 \mathrm{H},\left(\mathrm{CH}_{3}\right)_{2} \mathrm{CH}\right), 1.50$ (ddd,

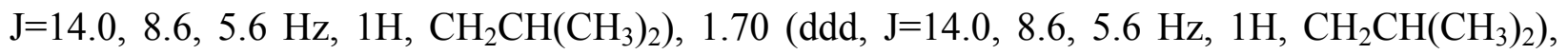
1.73-1.80 (m, 1H, $\left.\mathrm{CH}\left(\mathrm{CH}_{3}\right)_{2}\right), 3.42(\mathrm{dd}, \mathrm{J}=8.6,5.6 \mathrm{~Hz}, 1 \mathrm{H}, \mathrm{CHN}), 3.49(\mathrm{AB}, \mathrm{J}=13.6 \mathrm{~Hz}, 2 \mathrm{H}$, $\mathrm{NCH}_{2} \mathrm{Ph}$ ), 3.89 (AB system, J=13.6 Hz, 2H, $\mathrm{NCH}_{2} \mathrm{Ph}$ ), 5.14 (AB system, J=12.4 Hz, 1H, $\left.\mathrm{OCH}_{2} \mathrm{Ph}\right), 5.26\left(\mathrm{AB}, \mathrm{J}=12.4 \mathrm{~Hz}, 1 \mathrm{H}, \mathrm{OCH}_{2} \mathrm{Ph}\right), 7.18-7.44\left(\mathrm{~m}, 15 \mathrm{H}, \mathrm{H}_{\text {arom }}\right) .{ }^{13} \mathrm{C}$ NMR $(100 \mathrm{MHz}$, $\left.\mathrm{CDCl}_{3}\right) \delta 21.7\left(\left(\mathrm{CH}_{3}\right)_{2} \mathrm{CH}\right), 23.4\left(\left(\mathrm{CH}_{3}\right)_{2} \mathrm{CH}\right), 24.6\left(\mathrm{CH}\left(\mathrm{CH}_{3}\right)_{2}\right), 38.75\left(\mathrm{CH}_{2} \mathrm{CH}\left(\mathrm{CH}_{3}\right)_{2}, 54.6\right.$ $\left(\mathrm{NCH}_{2} \mathrm{Ph}\right), 58.9(\mathrm{CHN}), 66.1\left(\mathrm{OCH}_{2} \mathrm{Ph}\right), 127.1,128.3,128.4,128.6,128.8,129.1,136.3,139.8$, $173.4(\mathrm{C}=\mathrm{O})$.

(S)- $N$-Benzylleucine methyl ester 8. Benzyl bromide $(2.8 \mathrm{~g}, 2.0 \mathrm{~mL}, 17 \mathrm{mmol})$ was slowly added to a solution of L-leucine methyl ester hydrochloride $(3.0 \mathrm{~g}, 17 \mathrm{mmol})$ and $\mathrm{K}_{2} \mathrm{CO}_{3}(5.7 \mathrm{~g}$, $41 \mathrm{mmol})$ in acetonitrile $(40 \mathrm{~mL})$ at $0{ }^{\circ} \mathrm{C}$. The reaction mixture was stirred at room temperature for $12 \mathrm{~h}$. Then, water $(30 \mathrm{~mL})$ was added and the resulting mixture was extracted with ethyl acetate $(3 \times 40 \mathrm{~mL})$. The combined organic layers were dried over $\mathrm{Na}_{2} \mathrm{SO}_{4}$, filtered and evaporated under reduced pressure. The crude product was purified by flash chromatography using hexane:AcOEt $(10: 1)$ as eluent, to give 8 as a viscous liquid $2.9 \mathrm{~g}, 76 \%$ yield. $[\alpha]_{\mathrm{D}}=-42.3$ $\left(\mathrm{c}=1.3, \mathrm{CHCl}_{3}\right)$; Lit. $^{19}[\alpha]_{\mathrm{D}}=-40.7\left(\mathrm{c}=1.0, \mathrm{CHCl}_{3}\right)$.

Dimethyl (3S)-(3- $N, N$-dibenzylamino-5-methyl-2-oxohexylphosphonate $5 .{ }^{12}$ A solution of dimethyl methylphosphonate $(2.81 \mathrm{~g}, 22.7 \mathrm{mmol})$ in anhydrous THF $(40 \mathrm{~mL})$ was cooled at $78{ }^{\circ} \mathrm{C}$ before the slow addition of $n$-BuLi $2.5 \mathrm{M}$ in hexanes $(9.7 \mathrm{~mL}, 23.3 \mathrm{mmol})$. The resulting solution was stirred at $-50{ }^{\circ} \mathrm{C}$ for $1.5 \mathrm{~h}$ and then cooled to $-78{ }^{\circ} \mathrm{C}$. To this mixture was slowly added a solution of benzyl ester $4(2.6 \mathrm{~g}, 6.5 \mathrm{mmol})$ in dry THF $(45 \mathrm{~mL})$. The reaction mixture was stirred at $-78{ }^{\circ} \mathrm{C}$ for $4 \mathrm{~h}$ before the addition of a saturated solution of $\mathrm{NH}_{4} \mathrm{Cl}$. The solvent was evaporated under reduced pressure, the residue was dissolved in water $(30 \mathrm{~mL})$ and extracted with ethyl acetate $(3 \times 40 \mathrm{~mL})$. The combined organic extracts were dried over anhydrous $\mathrm{Na}_{2} \mathrm{SO}_{4}$, filtered and evaporated under reduced pressure. The crude product was purified by column chromatography using hexane:ethyl acetate (50:50) to give $\mathbf{5}$ as a viscous liquid $2.7 \mathrm{~g}, 98 \%$ yield. $[\alpha]_{\mathrm{D}}=-100\left(\mathrm{c}=3.78, \mathrm{CHCl}_{3}\right) .{ }^{1} \mathrm{H} \mathrm{NMR}\left(400 \mathrm{MHz}, \mathrm{CDCl}_{3}\right) \delta 0.80(\mathrm{~d}$, $\left.\mathrm{J}=6.6 \mathrm{~Hz}, 3 \mathrm{H},\left(\mathrm{CH}_{3}\right)_{2} \mathrm{CH}\right), 0.91\left(\mathrm{~d}, \mathrm{~J}=6.6 \mathrm{~Hz}, 3 \mathrm{H},\left(\mathrm{CH}_{3}\right)_{2} \mathrm{CH}\right), 1.34$ (ddd, J=13.0, 9.2, $3.2 \mathrm{~Hz}, 1 \mathrm{H}$, $\left.\mathrm{CH}_{2} \mathrm{CH}\left(\mathrm{CH}_{3}\right)_{2}\right)$, 1.41-1.51 (m, 1H, $\left.\mathrm{CH}\left(\mathrm{CH}_{3}\right)_{2}\right), 1.84$ (ddd, J=13.0, 9.2, $3.2 \mathrm{~Hz}, 1 \mathrm{H}$, $\mathrm{CH}_{2} \mathrm{CH}\left(\mathrm{CH}_{3}\right)_{2}$ ), 2.94 (dd, J=23.2, $14.0 \mathrm{~Hz}, 1 \mathrm{H}, \mathrm{CH}_{2} \mathrm{P}$ ), 3.42 (AB system, J=13.0 Hz, 2H, $\mathrm{CH}_{2} \mathrm{Ph}$ ), 3.45 (q, J=3.2 Hz, 1H, CHN), 3.48 (d, J=11.2 Hz, 3H, $\left.\left(\mathrm{CH}_{3} \mathrm{O}\right)_{2} \mathrm{P}\right), 3.64$ (dd, J=23.2, $\left.14.0 \mathrm{~Hz}, 1 \mathrm{H}, \mathrm{CH}_{2} \mathrm{P}\right), 3.65\left(\mathrm{~d}, \mathrm{~J}=11.2 \mathrm{~Hz}, 3 \mathrm{H},\left(\mathrm{CH}_{3} \mathrm{O}\right)_{2} \mathrm{P}\right), 3.68$ (AB system, J=13.0 Hz, 2H, $\left.\mathrm{CH}_{2} \mathrm{Ph}\right), 7.24-7.36\left(\mathrm{~m}, 10 \mathrm{H}, \mathrm{H}_{\text {arom }}\right){ }^{13} \mathrm{C} \mathrm{NMR}\left(100 \mathrm{MHz}, \mathrm{CDCl}_{3}\right) \delta 22.1\left(\left(\mathrm{CH}_{3}\right)_{2} \mathrm{CH}\right), 23.8$

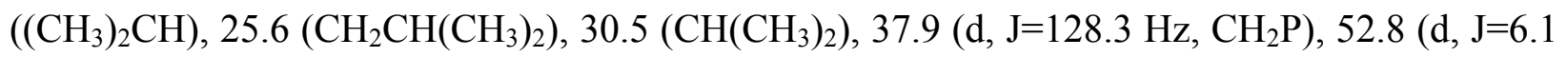
$\left.\mathrm{Hz},\left(\mathrm{CH}_{3} \mathrm{O}\right)_{2} \mathrm{P}\right), 52.9\left(\mathrm{~d}, \mathrm{~J}=6.1 \mathrm{~Hz},\left(\mathrm{CH}_{3} \mathrm{O}\right)_{2} \mathrm{P}\right), 54.7\left(\mathrm{CH}_{2} \mathrm{Ph}\right), 64.7\left(\mathrm{CHNBn}_{2}\right), 127.5,128.6$, 129.3, 139.3, $201.8(\mathrm{~d}, \mathrm{~J}=6.8 \mathrm{~Hz}, \mathrm{C}=\mathrm{O}) .{ }^{31} \mathrm{P} \mathrm{NMR}\left(200 \mathrm{MHz}, \mathrm{CDCl}_{3}\right) \delta 24.59$. Anal. calcd. for C23H32NO4P: C, 66.17; H, 7.61; N, 3.47\%. Found C, 65.93; H, 7.61; N, 3.47\%. 
Dimethyl (3S)- $N$-benzylamino-5-methyl-2-oxobuthylphosphonate 9. In an identical manner, the $(S)-N$-benzylleucine methyl ester $8(1.0 \mathrm{~g}, 4.2 \mathrm{mmol})$ in dry THF $(25 \mathrm{~mL})$ at $-78{ }^{\circ} \mathrm{C}$, was treated with a solution of dimethyl methylphosphonate $(1.6 \mathrm{~g}, 12.7 \mathrm{mmol})$ and $n$-BuLi $2.4 \mathrm{M}$ in hexanes $(5.5 \mathrm{~mL}, 13.2 \mathrm{mmol})$ in anhydrous THF $(25 \mathrm{~mL})$. The crude product $(74 \%)$ was analyzed by ${ }^{31} \mathrm{P}$ NMR $\left(200 \mathrm{MHz}, \mathrm{CDCl}_{3}\right) \delta 24.38$, and without any further purification was used in the next step.

Reduction of $\boldsymbol{\beta}$-ketophosphonate (S)-5 with $\mathbf{N a B H}_{4}$. To a solution of $\beta$-ketophosphonates $\mathbf{5}$ (1.0 equiv) in methanol $(20 \mathrm{~mL})$ cooled at $0^{\circ} \mathrm{C}$ was added $\mathrm{NaBH}_{4}$ (4.0 equiv). The reaction mixture was stirred at room temperature for $4 \mathrm{~h}$. The solvent was evaporated in vacuum, the residue was dissolved in water $(10 \mathrm{~mL})$ and extracted with ethyl acetate $(2 \mathrm{x} 20 \mathrm{~mL})$. The combined organic extracts were dried over anhydrous $\mathrm{Na}_{2} \mathrm{SO}_{4}$, filtered and concentrated in vacuum. The crude $\beta$-hydroxyphosphonates were analyzed by ${ }^{1} \mathrm{H} \mathrm{NMR}$ at $400 \mathrm{MHz}$ and ${ }^{31} \mathrm{P}$ NMR at $200 \mathrm{MHz}$, and then purified by flash chromatography using hexane:AcOEt (1:2) as eluent.

Dimethyl (3S)- $N$, $N$-dibenzylamino-(2R)-hydroxy-4-methylhexylphosphonate (syn-6). To a solution of $\beta$-ketophosphonate $5(1.0 \mathrm{~g}, 2.4 \mathrm{mmol})$ in dry THF $(40 \mathrm{~mL})$ cooled at $-20^{\circ} \mathrm{C}$ was added catecholborane $(9.6 \mathrm{~mL}, 9.6 \mathrm{mmol}) 1 \mathrm{M}$ in THF. The reaction mixture was stirred at $-78^{\circ}$ $\mathrm{C}$ for $4 \mathrm{~h}$, and quenched with a saturated solution of $\mathrm{NH}_{4} \mathrm{Cl}$. The solvent was evaporated in vacuum, the residue was dissolved in water $(30 \mathrm{~mL})$ and extracted with ethyl acetate $(3 \mathrm{x}$ $30 \mathrm{~mL}$ ). The combined organic extracts were dried over anhydrous $\mathrm{Na}_{2} \mathrm{SO}_{4}$, filtered and concentrated in vacuum. The crude $\beta$-hydroxyphosphonates were analyzed by ${ }^{1} \mathrm{H}$ NMR at 400 $\mathrm{MHz}$ and ${ }^{31} \mathrm{P}$ NMR at $200 \mathrm{MHz}$, and then purified by flash chromatography using hexane:AcOEt $(1: 2)$ as eluent to give as a viscous liquid $(871 \mathrm{mg}, 87 \%$ yield $)$ of syn-6. $[\alpha]_{\mathrm{D}}=-5.7$ (c $=2.9$, $\left.\mathrm{CHCl}_{3}\right) .{ }^{1} \mathrm{H} \mathrm{NMR}\left(400 \mathrm{MHz}, \mathrm{CDCl}_{3}\right) \delta 0.92\left(\mathrm{~d}, \mathrm{~J}=6.4 \mathrm{~Hz}, 3 \mathrm{H},\left(\mathrm{CH}_{3}\right)_{2} \mathrm{CH}\right), 0.93(\mathrm{~d}, \mathrm{~J}=6.4 \mathrm{~Hz}, 3 \mathrm{H}$, $\left(\mathrm{CH}_{3}\right)_{2} \mathrm{CH}$ ), 1.33 (ddd, J=13.6, 6.8, $\left.6.8 \mathrm{~Hz}, 1 \mathrm{H}, \mathrm{CH}_{2} \mathrm{CH}\left(\mathrm{CH}_{3}\right)_{2}\right), 1.58$ (ddd, J=13.6, 8.0, 4.8 Hz, $\left.1 \mathrm{H}, \mathrm{CH}_{2} \mathrm{CH}\left(\mathrm{CH}_{3}\right)_{2}\right), 1.69-1.72\left(\mathrm{~m}, 1 \mathrm{H}, \mathrm{CH}\left(\mathrm{CH}_{3}\right)_{2}\right), 1.77$ (ddd, J=20.0, 15.2, $\left.2.0 \mathrm{~Hz}, 1 \mathrm{H}, \mathrm{CH}_{2} \mathrm{P}\right)$, 1.95 (ddd, J=15.2, 15.2, $10.0 \mathrm{~Hz}, 1 \mathrm{H}, \mathrm{CH}_{2} \mathrm{P}$ ), 2.48 (ddd, J=8.0, 8.0, 4.8 Hz, 1H, CHNBn $), 3.43$ ( $\mathrm{AB}$ system, $\left.\mathrm{J}=13.4 \mathrm{~Hz}, 2 \mathrm{H}, \mathrm{CH}_{2} \mathrm{Ph}\right), 3.71\left(\mathrm{~d}, \mathrm{~J}=11.0 \mathrm{~Hz}, 3 \mathrm{H},\left(\mathrm{CH}_{3} \mathrm{O}\right)_{2} \mathrm{P}\right), 3.75(\mathrm{~d}, \mathrm{~J}=11.0 \mathrm{~Hz}$, $\left.3 \mathrm{H},\left(\mathrm{CH}_{3} \mathrm{O}\right)_{2} \mathrm{P}\right), 3.86-3.92(\mathrm{~m}, 1 \mathrm{H}, \mathrm{CHOH}), 3.92$ (AB system, J=13.4 Hz, 2H, $\left.\mathrm{CH}_{2} \mathrm{Ph}\right), 7.21-7.32$ $\left(\mathrm{m}, 10 \mathrm{H}, \mathrm{H}_{\text {arom }}\right) .{ }^{13} \mathrm{C} \mathrm{NMR}\left(100 \mathrm{MHz}, \mathrm{CDCl}_{3}\right) \delta 23.0\left(\left(\mathrm{CH}_{3}\right)_{2} \mathrm{CH}\right), 23.6\left(\left(\mathrm{CH}_{3}\right)_{2} \mathrm{CH}\right), 26.4$ $\left(\mathrm{CH}_{2} \mathrm{CH}\left(\mathrm{CH}_{3}\right)_{2}\right), 30.8\left(\mathrm{~J}=140.4 \mathrm{~Hz}, \mathrm{CH}_{2} \mathrm{P}\right), 34.9\left(\mathrm{CH}\left(\mathrm{CH}_{3}\right)_{2}\right), 52.5\left(\mathrm{~d}, \mathrm{~J}=6.6 \mathrm{~Hz},\left(\mathrm{CH}_{3} \mathrm{O}\right)_{2} \mathrm{P}\right), 52.8$ $\left(\mathrm{d}, \mathrm{J}=6.6 \mathrm{~Hz},\left(\mathrm{CH}_{3} \mathrm{O}\right)_{2} \mathrm{P}\right), 54.6\left(\mathrm{CH}_{2} \mathrm{Ph}\right), 60.8(\mathrm{~d}, \mathrm{~J}=18.2 \mathrm{~Hz}, \mathrm{CHOH}), 67.3(\mathrm{~d}, \mathrm{~J}=6.1 \mathrm{~Hz}$, $\mathrm{CHNBn}_{2}$ ), 127.3, 128.6, 129.3, 139.7. ${ }^{31} \mathrm{P} \mathrm{NMR}\left(200 \mathrm{MHz}, \mathrm{CDCl}_{3}\right) \delta$ 34.72. Anal. calcd. for C23H34NO4P: C, 65.85; H, 8.17; N, 3.34\%. Found C, 65.47; H, 8.04; N, 3.21\%.

Dimethyl (3S)- $N$-benzylamino-(2S)-hydroxy-4-methylhexylphosphonate (anti-10). In an identical manner the 2-ketophosphonate $9(1.4 \mathrm{~g}, 4.2 \mathrm{mmol})$ in dry THF $(20 \mathrm{~mL})$ was treated a $78^{\circ} \mathrm{C}$ with a solution of $\mathrm{Zn}\left(\mathrm{BH}_{4}\right)_{2}(77 \mathrm{~mL}, 17 \mathrm{mmol}) 1 \mathrm{M}$ in THF, ${ }^{20}$ obtaining the mixture of 2hydroxyphosphonates anti-10 and syn-11 in a 96:4 ratio, respectively. The mixture was purified by flash chromatography obtaining the 2-hydroxyphosphonate anti-10 diastereomerically pure as a viscous liquid $(1.3 \mathrm{~g}, 95 \%$ yield $) .[\alpha]_{\mathrm{D}}=-9.9\left(\mathrm{c}=2.2, \mathrm{CHCl}_{3}\right) .{ }^{1} \mathrm{H} \mathrm{NMR}\left(400 \mathrm{MHz}, \mathrm{CDCl}_{3}\right)$ 
$\delta 0.81\left(\mathrm{~d}, \mathrm{~J}=6.8 \mathrm{~Hz}, 3 \mathrm{H},\left(\mathrm{CH}_{3}\right)_{2} \mathrm{CH}\right), 0.91\left(\mathrm{~d}, \mathrm{~J}=6.8 \mathrm{~Hz}, 3 \mathrm{H},\left(\mathrm{CH}_{3}\right)_{2} \mathrm{CH}\right), 1.16(\mathrm{ddd}, \mathrm{J}=14.0,8.8$, $\left.4.8 \mathrm{~Hz}, 1 \mathrm{H}, \mathrm{CH}_{2} \mathrm{CH}\left(\mathrm{CH}_{3}\right)_{2}\right), 1.33$ (ddd, J=14.0, 8.8, $\left.5.6 \mathrm{~Hz}, 1 \mathrm{H}, \mathrm{CH}_{2} \mathrm{CH}\left(\mathrm{CH}_{3}\right)_{2}\right)$, 1.56-1.66 (m, $\left.1 \mathrm{H}, \mathrm{CH}\left(\mathrm{CH}_{3}\right)_{2}\right), 1.87$ (ddd, J=19.6, 15.2, 3.2 Hz, 1H, $\mathrm{CH}_{2} \mathrm{P}$ ), 2.00 (ddd, J=16.8, 15.2, $10.0 \mathrm{~Hz}$, $\left.1 \mathrm{H}, \mathrm{CH}_{2} \mathrm{P}\right), 2.70$ (ddd, J=8.8, 8,8, $\left.4.4 \mathrm{~Hz}, 1 \mathrm{H}, \mathrm{CHNH}\right), 3.76\left(\mathrm{~d}, \mathrm{~J}=10.8 \mathrm{~Hz}, 3 \mathrm{H},\left(\mathrm{CH}_{3} \mathrm{O}\right)_{2} \mathrm{P}\right), 3.77$ ( $\mathrm{AB}$ system, $\left.\mathrm{J}=13.2 \mathrm{~Hz}, 1 \mathrm{H}, \mathrm{CH}_{2} \mathrm{Ph}\right), 3.78\left(\mathrm{~d}, \mathrm{~J}=10.8 \mathrm{~Hz}, 3 \mathrm{H},\left(\mathrm{CH}_{3} \mathrm{O}\right)_{2} \mathrm{P}\right), 3.86$ (AB system, $\left.\mathrm{J}=13.2 \mathrm{~Hz}, 1 \mathrm{H}, \mathrm{CH}_{2} \mathrm{Ph}\right), 4.10-4.16(\mathrm{~m}, 1 \mathrm{H}, \mathrm{CHOH}), 7.23-7.35\left(\mathrm{~m}, 5 \mathrm{H}, \mathrm{H}_{\text {arom}}\right) .{ }^{13} \mathrm{C} \mathrm{NMR}(100$ $\left.\mathrm{MHz}, \mathrm{CDCl}_{3}\right) \delta 22.5\left(\left(\mathrm{CH}_{3}\right)_{2} \mathrm{CH}\right), 23.6\left(\left(\mathrm{CH}_{3}\right)_{2} \mathrm{CH}\right), 24.9\left(\mathrm{CH}_{2} \mathrm{CH}\left(\mathrm{CH}_{3}\right)_{2}\right), 28.1(\mathrm{~J}=141.2 \mathrm{~Hz}$, $\left.\mathrm{CH}_{2} \mathrm{P}\right), 38.6\left(\mathrm{CH}\left(\mathrm{CH}_{3}\right)_{2}\right), 52.1\left(\mathrm{CH}_{2} \mathrm{Ph}\right), 52.4\left(\mathrm{~d}, \mathrm{~J}=7.6 \mathrm{~Hz},\left(\mathrm{CH}_{3} \mathrm{O}\right)_{2} \mathrm{P}\right), 52.8(\mathrm{~d}, \mathrm{~J}=7.6 \mathrm{~Hz}$, $\left.\left(\mathrm{CH}_{3} \mathrm{O}\right)_{2} \mathrm{P}\right), 59.2(\mathrm{~d}, \mathrm{~J}=15.2 \mathrm{~Hz}, \mathrm{CHOH}), 66.5(\mathrm{CHNH}), 127.3,128.4,128.6,140.4 .{ }^{31} \mathrm{P}$ NMR $\left(200 \mathrm{MHz}, \mathrm{CDCl}_{3}\right) \delta 35.03$. HRMS $\left(\mathrm{CI}^{+}, \mathrm{CH}_{4}\right)$ calcd. for $\mathrm{C}_{16} \mathrm{H}_{29} \mathrm{NO}_{4} \mathrm{P}\left(\mathrm{MH}^{+}\right) 330.1756$ found 330.1519.

(3S)-Amino-(2R)-hydroxy-5-methylhexylphosphonic acid (phosphostatine) (12). 2-Hydroxyphosphonate syn-6 (450 mg, $1.07 \mathrm{mmol})$ was treated at $0{ }^{\circ} \mathrm{C}$ under a nitrogen atmosphere with bromotrimethylsilane $(361 \mathrm{mg}, 0.31 \mathrm{~mL}, 2.36 \mathrm{mmol})$. The reaction mixture was stirred at room temperature for 6-8 h, and after this period of time the volatile materials were evaporated under reduced pressure, water was then added. After $30 \mathrm{~min}$. the solvents were evaporated in vacuo to give (3S)- $N, N$-dibenzylamino-(2R)-hydroxyphosphonic acid, which without isolation were treated with palladium on carbon $(5 \% \mathrm{wt}) 225 \mathrm{mg}$ in methanol $(10 \mathrm{~mL})$ and stirred for $12 \mathrm{~h}$ under a hydrogen gas atmosphere at room temperature. The mixture was filtered through a pad of celite, and the solvent was evaporated under reduced pressure. The residue was treated with propylene oxide $(5 \mathrm{~mL})$ to give the title compound as a white solid (201 mg, 89\% yield). m.p. 220-222 ${ }^{\circ} \mathrm{C} .[\alpha]_{\mathrm{D}}=-11.4\left(\mathrm{c}=2.76, \mathrm{H}_{2} \mathrm{O}\right) .{ }^{1} \mathrm{H} \mathrm{NMR}\left(400 \mathrm{MHz}, \mathrm{D}_{2} \mathrm{O}\right) \delta 0.93(\mathrm{~d}, \mathrm{~J}=6.4 \mathrm{~Hz}, 3 \mathrm{H}$, $\left.\left(\mathrm{CH}_{3}\right)_{2} \mathrm{CH}\right), 0.95\left(\mathrm{~d}, \mathrm{~J}=6.4 \mathrm{~Hz}, 3 \mathrm{H},\left(\mathrm{CH}_{3}\right)_{2} \mathrm{CH}\right), 1.51$ (ddd, J=14.4, 6.4, $6.4 \mathrm{~Hz}, 1 \mathrm{H}$, $\mathrm{CH}_{2} \mathrm{CH}\left(\mathrm{CH}_{3}\right)_{2}$ ), 1.56 (ddd, J=14.4, 6.4, $\left.6.4 \mathrm{~Hz}, 1 \mathrm{H}, \mathrm{CH}_{2} \mathrm{CH}\left(\mathrm{CH}_{3}\right)_{2}\right), 1.71$ (ninth, J=6.4 Hz, 1H, $\left.\mathrm{CH}\left(\mathrm{CH}_{3}\right)_{2}\right), 1.87$ (ddd, J=17.6, 15.2, 8.0 Hz, 1H, $\left.\mathrm{CH}_{2} \mathrm{P}\right), 1.96$ (ddd, J=19.6, 15.2, $4.8 \mathrm{~Hz}, 1 \mathrm{H}$, $\left.\mathrm{CH}_{2} \mathrm{P}\right), 3.38$ (dt, J=8.4, $\left.5.2 \mathrm{~Hz}, 1 \mathrm{H}, \mathrm{CHNH}_{2}\right), 4.00-4.07$ (m, 1H, CHOH). ${ }^{13} \mathrm{C} \mathrm{NMR}(100 \mathrm{MHz}$, $\left.\mathrm{D}_{2} \mathrm{O}\right) \delta 23.6\left(\left(\mathrm{CH}_{3}\right)_{2} \mathrm{CH}\right), 24.7\left(\left(\mathrm{CH}_{3}\right)_{2} \mathrm{CH}\right), 26.4\left(\mathrm{CH}_{2} \mathrm{CH}\left(\mathrm{CH}_{3}\right)_{2}\right), 34.8\left(\mathrm{~J}=130.5 \mathrm{~Hz}, \mathrm{CH}_{2} \mathrm{P}\right), 40.9$ $\left(\mathrm{CH}\left(\mathrm{CH}_{3}\right)_{2}\right), 57.0$ (d, J=12.1 Hz, $\left.\mathrm{CHOH}\right), 69.4$ (d, J=3.1 Hz, $\left.\mathrm{CHNH}_{2}\right) .{ }^{31} \mathrm{P} \mathrm{NMR}(200 \mathrm{MHz}$, $\left.\mathrm{D}_{2} \mathrm{O}\right) \delta$ 21.10. HRMS $\left(\mathrm{CI}^{+}, \mathrm{CH}_{4}\right)$ calcd. for $\mathrm{C}_{7} \mathrm{H}_{19} \mathrm{NO}_{4} \mathrm{P}\left(\mathrm{MH}^{+}\right) 212.1959$ found 212.1906.

(3S)-Amino-(2S)-hydroxy-5-methylhexylphosphonic acid (phosphoepistatine) (13). In an identical manner, the 2-hydroxyphosphonate anti-10 (350 mg, $1.06 \mathrm{mmol})$ was treated with bromotrimethylsilane $(357 \mathrm{mg}, 0.31 \mathrm{~mL}, 2.33 \mathrm{mmol})$ followed by palladium on carbon (10\% wt) $(175 \mathrm{mg})$ in methanol $(10 \mathrm{~mL})$ and propylene oxide $(5 \mathrm{~mL})$ to give the title compound as a white solid (208 mg, 93\% yield). m.p. $224-226{ }^{\circ} \mathrm{C}$. $[\alpha]_{\mathrm{D}}=-20.8,\left(\mathrm{c}=1.81, \mathrm{H}_{2} \mathrm{O}\right) .{ }^{1} \mathrm{H}$ NMR $(400$ $\left.\mathrm{MHz}, \mathrm{D}_{2} \mathrm{O}\right) \delta 0.92\left(\mathrm{~d}, \mathrm{~J}=6.2 \mathrm{~Hz}, 3 \mathrm{H},\left(\mathrm{CH}_{3}\right)_{2} \mathrm{CH}\right), 0.97\left(\mathrm{~d}, \mathrm{~J}=6.2 \mathrm{~Hz}, 3 \mathrm{H},\left(\mathrm{CH}_{3}\right)_{2} \mathrm{CH}\right), 1.46$ (ddd, $\left.\mathrm{J}=14.4,10.0,4.4 \mathrm{~Hz}, 1 \mathrm{H}, \mathrm{CH}_{2} \mathrm{CH}\left(\mathrm{CH}_{3}\right)_{2}\right), 1.56$ (ddd, J=14.4, 10.0, $\left.4.4 \mathrm{~Hz}, 1 \mathrm{H}, \mathrm{CH}_{2} \mathrm{CH}\left(\mathrm{CH}_{3}\right)_{2}\right)$, 1.62-1.72 (m, 1H, $\left.\mathrm{CH}\left(\mathrm{CH}_{3}\right)_{2}\right), 1.79$ (ddd, J=18.0, 15.2, $\left.8.4 \mathrm{~Hz}, 1 \mathrm{H}, \mathrm{CH}_{2} \mathrm{P}\right), 1.87$ (ddd, J=18.8, $\left.15.2,5.6 \mathrm{~Hz}, 1 \mathrm{H}, \mathrm{CH}_{2} \mathrm{P}\right), 3.51\left(\mathrm{ddd}, \mathrm{J}=9.6,4.0,4.0 \mathrm{~Hz}, 1 \mathrm{H}, \mathrm{CHNH}_{2}\right), 4.17-4.24(\mathrm{~m}, 1 \mathrm{H}, \mathrm{CHOH})$. ${ }^{13} \mathrm{C}$ NMR $\left(100 \mathrm{MHz}, \mathrm{D}_{2} \mathrm{O}\right) \delta 23.1\left(\left(\mathrm{CH}_{3}\right)_{2} \mathrm{CH}\right), 25.2\left(\left(\mathrm{CH}_{3}\right)_{2} \mathrm{CH}\right), 26.3\left(\mathrm{CH}_{2} \mathrm{CH}\left(\mathrm{CH}_{3}\right)_{2}\right), 33.4$ $\left(\mathrm{J}=130.6 \mathrm{~Hz}, \mathrm{CH}_{2} \mathrm{P}\right), 37.7\left(\mathrm{CH}\left(\mathrm{CH}_{3}\right)_{2}\right), 56.5(\mathrm{~d}, \mathrm{~J}=10.6 \mathrm{~Hz}, \mathrm{CHOH}), 70.1\left(\mathrm{~d}, \mathrm{~J}=3.1 \mathrm{~Hz}, \mathrm{CHNH}_{2}\right)$. 
${ }^{31} \mathrm{P}$ NMR $\left(200 \mathrm{MHz}, \mathrm{D}_{2} \mathrm{O}\right) \delta$ 21.14. HRMS $\left(\mathrm{CI}^{+}, \mathrm{CH}_{4}\right)$ calcd. for $\mathrm{C}_{7} \mathrm{H}_{19} \mathrm{NO}_{4} \mathrm{P}\left(\mathrm{MH}^{+}\right) 212.1959$ found 212.1884 .

\section{Acknowledgements}

The authors are grateful to the CONACYT of Mexico via grant 41657-Q and UQUIFA-México for financial support of this work. We also thank Victoria Labastida for her valuable technical help for the MS spectra and Citlali Quiñónes for some preparation and preliminary experiments.

\section{References and footnotes}

1. (a) Aoyagi, T.; Morishima, H.; Nishizawa, R.; Kunimoto, S.; Takeuchi, T.; Umezawa, H.; Ikezawa, H. J. Antibiot. 1972, 25, 689. (b) Umezawa, H.; Aoyagi, T.; Morishima, H.; Matsuzaki, M.; Hamada, M.; Takeuchi, T. J. Antibiot. 1970, 23, 259.

2. For recent synthetic methodologies of statine and their analogues, see: (a) Yuste, F.; Diaz, A.; Ortiz, B.; Sánchez-Obregón, R.; Walls, F.; García-Ruano, J. L. Tetrahedron: Asymmetry 2003, 14, 549. (b) Yoo, D.; Oh, J. S.; Kim, Y. G. Org. Lett. 2002, 4, 1213. (c) Kwon, S. J.; Ko, S. Y. Tetrahedron Lett. 2002, 43, 639. (d) Ko, S. Y. J. Org. Chem. 2002, 67, 2689. (e) Pasenti, C.; Bravo, P.; Corradi, E.; Frigerio, M.; Meille, S. V.; Panzeri, W.; Viani, F.; Zanda, M. J. Org. Chem. 2001, 66, 5637. (f) Travins, J. M.; Bursavich, M. G.; Veber, D. F.; Rich, D. H. Org. Lett. 2001, 3, 2725. (g) Hoffman, R. V.; Tao, J. J. Org. Chem. 1997, 62, 2292. (h) Sengupta, S.; Sarma, D. S. Tetrahedron: Asymmetry 1999, 10, 4633. (i) Lee, K.-Y.; Kim, H.-Y.; Park, M.-S.; Oh, C.-Y.; Ham, W.-H. J. Org. Chem. 1999, 64, 9450. (j) Alemay, C.; Bach, J.; Farras, J.; García, J. Org. Lett. 1999, 1, 1831. (k) Reddy, G. V.; Rao, G. V.; Iyengar, D. S. Tetrahedron Lett. 1999, 40, 775. (1) Aoyagi, Y.; Williams, R. M. Tetrahedron 1998, 54, 10419. (m) Veeresha, G.; Datta, A. Tetrahedron Lett. 1997, 38, 5223. (n) Gennari, C.; Moresca, D.; Vulpetti, A.; Pain, G. Tetrahedron 1997, 53, 5593. (o) Ma, D.; Ma, J.; Ding, W.; Dai, L. Tetrahedron: Asymmetry 1996, 7, 2365.

3. Aminophosphonic and Aminophosphinic Acids: Chemistry and Biological Activity. Kukhar, V. P.; Hudson H. R., Eds; John Wiley: New York, 2000 and references therein.

4. (a) Nieschalk, J.; Batsanov, A. S.; O’Hagan, D.; Howard, J. A. K. Tetrahedron 1996, 52, 165. (b) Burke Jr. T. R.; Smyth, M. S.; Nomizu, M.; Otaka, A.; Roller, P. P. J. Org. Chem. 1993, 58, 1336.

5. (a) Dellaria, J. F., Jr.; Maki, R. G.; Stein, H. H.; Cohen, J.; Whittern, D.; Marsh, K.; Hoffman, D. J.; Plattner, J. J.; Perum, T. J. J. Med. Chem. 1990, 33, 534. (b) Dellaria, J. F.; Maki, R. G. Tetrahedron Lett. 1986, 27, 2337.

6. Chakravarty, P. K.; Greenlee, W. J.; Parsons, W. H.; Patchett, A. A.; Combs, P.; Roth, A.; Busch, R. D.; Melllin, T. N. J. Med. Chem. 1989, 32, 1886.

7. (a) Drag, M.; Latjka, R.; Gumienna-Kontecka, E.; Kozlowski, H.; Kafarski, P. Tetrahedron: Asymmetry 2003, 14, 1837. (b) Wróblewski, A. E.; Piotrowska, D. G. Tetrahedron: 
Asymmetry 2002, 13, 2509 and references therein. (c) Hammerschmidt, F.; Wolfgang, W.; Wuggenig, F.; Zarbl, E. Tetrahedron: Asymmetry 2000, 11, 2955. (d) Hammerschmidt, F.; Lindner, W.; Wuggenig, F.; Zarbl, E. Tetrahedron: Asymmetry 2000, 11, 2955. (e) Barco, A.; Benetti, S.; Bergamini, P.; De Risi, C.; Marchetti, P.; Pollini, G. P.; Zanirato, V. Tetrahedron Lett. 1999, 40, 7705. (f) Thomas, A. A.; Sharpless, K. B. J. Org. Chem. 1999, 64, 8379. (g) Gravotto, G.; Giovenzana, G. B.; Pagliarin, R.; Palmisano, G.; Sisti, M. Tetrahedron: Asymmetry 1998, 9, 745.

8. Yamagishi, T.; Fujii, K.; Shibuya, S.; Yokomatsu, T. Synlett 2004, 2505.

9. (a) Ordóñez, M.; González-Morales, A.; Ruiz, C.; De la Cruz-Cordero, R.; FernándezZertuche, M. Tetrahedron: Asymmetry 2003, 14, 1775. (b) Ordóñez, M.; González-Morales, A.; Salazar-Fernández, H. Tetrahedron: Asymmetry 2004, 15, 2719.

10. Reduction of other 2-ketophosphonates have been described by us, see: Ordóñez, M.; De la Cruz-Cordero, R.; Fernández-Zertuche, M.; Muñoz-Hernández, M. A. Tetrahedron: Asymmetry 2002, 13, 559.

11. The preparation of ester $\mathbf{4}$ has been described, although to the best of our knowledge no data given: (a) Reetz, M. T.; Drewes, M. W.; Schmitz, A. Angew. Chem., Int. Ed. 1987, 26, 1141. (b) Cooke, J. W. B.; Davies, S. G.; Naylon, A. Tetrahedron 1993, 49, 7955. (c) Beaulieu, P. L.; Wernic, D. J. Org. Chem. 1996, 61, 3635. (d) Chung, S.-K.; Kang, D.-H. Tetrahedron: Asymmetry 1997, 8, 3027.

12. The preparation of 2-ketophosphonate $\mathbf{5}$ has been described, although to the best of our knowledge no data given: see ref. $11 \mathrm{~d}$.

13. (a) Cherest, M.; Felkin, H. Tetrahedron Lett. 1968, 18, 2199. (b) Cherest, M.; Felkin, H. Tetrahedron Lett. 1968, 18, 2205. (c) Anh, N. T.; Eisenstein, O. Nouv J. Chem. 1977, 1, 61. (d) For an excellent summary, see: Eliel, E. L.; Wilen, S. H.; Mander, L. N. Stereochemistry of Organic Compounds; John Wiley and Sons: New York, 1994; pp 876.

14. Barluenga, J.; Baragaña, B.; Concellón, J. M. J. Org. Chem. 1995, 60, 6696.

15. (a) Hoffmann, R. V.; Maslouh, N.; Cervantes-Lee, F. J. Org. Chem. 2002, 67, 1045. (b) Reetz, M. T.; Schmitz, A. Tetrahedron Lett. 1999, 40, 2741.

16. Concellón, J. M.; Bernard, P. L.; Riego, E.; García-Granda, S.; Forcén-Acebal, A. J. Org. Chem. 2001, 66, 2764.

17. Preparation of 2-ketophosphonate 9 and dimethyl (3S)- $N$-benzylamino-(2S)-hydroxy-4methylhexylphosphonate anti-10 has been reported by us, but no data given: Ordóñez. M.; De la Cruz-Cordero, R.; Quiñónes, C.; González-Morales, A. J. Chem. Soc., Chem. Commun. 2004, 672.

18. Chung McKenna, C. E.; Higa, M. T.; Cheung, N. H.; McKenna, M.-C. Tetrahedron Lett. 1977, 155.

19. Laschat, S.; Frohlinch, R.; Wibbeling, B. J. Org. Chem. 1996, 61, 2829.

20. For the preparation of $\mathrm{Zn}\left(\mathrm{BH}_{4}\right)_{2}$ see: Pelter, A.; Smith, K.; Brown, H. Borane Reagents, Academic Press: London, 1988; p 414. 